\title{
Raman study of
}

\section{2,7-bis(biphenyl-4-yl-)2',7'-ditertbutyl-9,9'-spirobifluorene}

\section{adsorbed on oxide surfaces}

Julia Stähler ${ }^{1,2}$, Oliver T. Hofmann ${ }^{1}$, Patrick Rinke ${ }^{1}$, Sylke Blumstengel ${ }^{3}$, Fritz Henneberger ${ }^{3}$, Yilei Li $^{2}$, Tony F. Heinz ${ }^{2}$

${ }^{1}$ Fritz-Haber-Institut der Max-Planck-Gesellschaft, Faradayweg 4-6, 14195 Berlin, Germany

${ }^{2}$ Departments of Physics and Electrical Engineering, Columbia University, 538 West 120th Street, New York, New York 10027, USA

${ }^{3}$ Institut für Physik, Humboldt-Universität zu Berlin, Newtonstrasse 15, 12489 Berlin, Germany

Keywords: non-resonant Raman; inorganic/organic hybrid; optoelectronics

\section{Highlights}

- $\quad$ substrate influence on the non-resonant Raman response of SP6 molecules

- $\quad$ three substrates and various SP6 layer thicknesses investigated

- Raman intensity of SP6 monolayer comparable to substrate signal: high cross section

- comparison of the Raman spectra to gas phase DFT calculations

- no significant electronic perturbation of SP6 on all of the investigated surfaces

\begin{abstract}
We present a systematic non-resonant Raman study of the vibrational properties of the spirobifluorene derivative 2,7-bis(biphenyl-4-yl)-2',7'-di-tertbutyl-9,9'-spirobifluorene (SP6) on three different substrates: $\mathrm{ZnO}(000-1), \mathrm{ZnMgO}(000-1)$, and $\mathrm{Al}_{2} \mathrm{O}_{3}(11-20)$. This investigation of few monolayer coverages is facilitated by the high scattering cross section of this molecule. The vibrational response of SP6 is unaffected by the choice of substrate, showing that the vibrational properties of the molecules remain unchanged by adsorption. Furthermore, we can directly compare the experimental spectra to our density functional theory calculations of the Raman cross sections of SP6 in the gas phase.
\end{abstract}




\section{Introduction}

Organic/inorganic semiconductor hybrid structures hold the promise to unite specific complementary properties of both material classes leading to optoelectronic and photonic devices with advanced functionalities. ${ }^{1}$ Interface formation between these two vastly different materials is a very complex issue. Inorganic semiconductor surfaces present dangling bonds, steps, and surface defects. Binding of molecules on such sites can cause modifications of their electronic structure or even their fragmentation resulting in ill-defined interfaces. To exploit the potential of organic/inorganic hybrid structures, control over the structural and electronic properties of the heterointerface would be highly beneficial.

The high fluorescence quantum yield and absorption cross section of 2,7-bis(biphenyl-4-yl)-2',7'-di-tertbutyl-9,9'-spirobi-fluorene (SP6) as well as the excellent charge carrier injection and transport properties of the $\mathrm{ZnO}(000-1)$ and $\mathrm{ZnMgO}(000-1)$ make combined systems very promising candidates for hybrid light-emitting or photovoltaic devices. In previous works, it was established that both excitons and charges are transferred with high efficiency across the heterointerface of $\mathrm{SP} 6$ and $\mathrm{ZnO} / \mathrm{ZnMgO}$ quantum structures. ${ }^{2}$ The electronic structure of this interface was probed with photoemission spectroscopy, and no evidence of modification of the electronic structure of the molecule was found.

In this article, we study the vibrational properties of such organic/inorganic hybrid structures by nonresonant Raman spectroscopy. Whether or not conformational changes of adsorbed molecules should lead to changes in the Raman response compared to the ensemble of molecules in thick films is a controversially discussed issue (see, for example Refs. $[3,4,5,6]$ ). However, line shifts, and changes of Raman intensities and line widths have been observed for surface-enhanced Raman spectroscopy of adsorbed molecules on surfaces. ${ }^{7,8}$ A significant perturbation of the electronic wave functions due to interactions of the molecules with the semiconductor surface should lead to a frequency shift of the vibrational modes. Also, geometry changes with respect to the gas phase (as, for instance, the dihedral angle of the biphenyl) can have a strong influence on the Raman response of the molecules. Still (nonenhanced) Raman spectroscopy of adsorbed molecules suffers from low intensities, which is the reason for the great success of tip- and surface-enhanced experiments. We present non-resonant and non- 
enhanced Raman spectroscopy of SP6 on the polar $\mathrm{ZnO}(000-1)$ and $\mathrm{ZnMgO}(000-1)$ surface and compare it to spectra of similar films on $\mathrm{Al}_{2} \mathrm{O}_{3}(11-20)$ substrates to identify potential substrate-adsorbate interactions. This study is made possible by the very high scattering cross section of the investigated molecule SP6 that leads to Raman intensities on the order of the bulk substrate signal even for monolayer coverages. We also vary the thickness of the organic layer from one monolayer up to $16.0 \mathrm{~nm}$ thick layers and compare the spectra with calculated vibrational modes of the isolated SP6 molecule calculated by density functional theory (DFT). Despite the fact that these calculations were performed for the gas phase, the result matches the experimental spectra very well, suggesting the identification of the different Raman modes in this large molecule. Experimentally, we do not find any shift of the vibrational frequencies with increasing organic layer thickness and observe a linear dependence of the Raman intensities with the number of molecules. The good agreement between experiment and calculation also confirms that the interaction of SP6 with the $\mathrm{ZnO}(000-1)$ and $\mathrm{ZnMgO}(000-1)$ surface is weak and shows that the molecules adsorb without strong electronic interactions on these semiconductor surfaces.

\section{Details of experiment and calculation}

The samples are prepared in a molecular beam epitaxy apparatus (DCA450) equipped with separate growth chambers for $(\mathrm{Zn}, \mathrm{Mg}) \mathrm{O}$ and organic molecules. The background pressure is $10^{-10} \mathrm{Torr}$. $\mathrm{ZnO}(000-$ 1) and $\mathrm{Zn}_{1-\mathrm{x}} \mathrm{Mg}_{\mathrm{x}} \mathrm{O}(x=0.14)$ epilayers (thickness $\left.500 \mathrm{~nm}\right)$ are grown on $a$-plane sapphire substrates by radical-source MBE, resulting in the oxygen-terminated (000-1) surface. Details on the growth have been published elsewhere. ${ }^{9}$ Once the inorganic layers have been prepared, the samples are transferred under ultra-high vacuum conditions to the growth chamber for organic molecules. Retaining the sample under ultrahigh vacuum ensures the presence of well-defined interfaces of the highest purity. The SP6 molecules were purchased from Merck and used without further purification. The molecules are sublimed from Knudson-type effusion cells. The thickness of the deposited layer is monitored by a quartz microbalance. The deposition rate was $0.1 \mathrm{~nm} / \mathrm{min}$ and the substrate was kept at room temperature during 
growth. It was established previously by atomic force microscopy that SP6 forms homogenous, amorphous thin films on $\mathrm{ZnO}(000-1)$ and $\mathrm{ZnMgO}(000-1)$ surfaces. $^{2}$

For Raman spectroscopy, we use a continuous wave laser at $532 \mathrm{~nm}$, which is focused using a 50x objective lens $(\mathrm{NA}=0.75)$ onto our samples. As the optical absorption of the samples begins in the ultraviolet range $(\mathrm{h} v>3.4 \mathrm{eV}){ }^{2}$ Raman scattering surely occurs non-resonantly. The incident optical power is $1.0 \mathrm{~mW}$, with a spot size of $0.5 \mu \mathrm{m}$ on the sample. The Raman signal from the sample is collected by the objective and dispersed onto a thermoelectrically cooled CCD camera using a grating with a groove density of $1800 \mathrm{~cm}^{-1}$. The Raman spectra are obtained with an integration time of $300 \mathrm{~s}$. Multiple spectra are taken at different locations on each sample leading to almost identical spectra (not shown) and confirming the uniformity of the samples. All spectra shown are raw data, corrected only for background by the subtraction of a single Gaussian feature wider than $1500 \mathrm{~cm}^{-1}$.

We performed the density functional theory calculations with the Gaussian09 package ${ }^{10}$ using the B3LYP exchange-correlation functional together with a $6-31 \mathrm{G}^{*}$ basis set. The integration grids were set to "ultrafine". The vibrational modes were calculated within the harmonic approximation. Scott and Radom reported an RMS error of $34 \mathrm{~cm}^{-1}$ for this methodology and suggested scaling the frequencies by a universal factor of $0.9614,{ }^{11}$ which we have done throughout. All calculations were performed for a single molecule in the gas phase. Therefore, geometrical changes induced by condensation into the solid state, such as reductions of the biphenyl dihedral angle, are not captured within the calculations. Furthermore, the harmonic approximation of the vibrations yields line spectra only. For this reason, as well as because of the neglect of disorder in the films and of finite temperature effects, we do not obtain finite line widths that could be compared to the experimental Raman features. For this purpose, the calculated spectra shown in this work are broadened by $2 \mathrm{~cm}^{-1}$.

\section{Results}

Fig. 1 depicts a series of non-resonant ${ }^{12}$ Raman spectra for different coverages of SP6 on sapphire between 800 and $1700 \mathrm{~cm}^{-1} .{ }^{13}$ The spectra are dominated by a strong mode at $1603 \mathrm{~cm}^{-1}$ and exhibit 
several features above $800 \mathrm{~cm}^{-1}$, including a broad band around $1300 \mathrm{~cm}^{-1}$. In Table 1 we compare these spectral signatures to the literature dealing with related molecules, such as oligophenyls ${ }^{14,15}$, octylfluorene oligomers ${ }^{16}$, and 9,9-spirobifluorene ${ }^{17}$. This comparison suggests that the modes around $1600 \mathrm{~cm}^{-1}$ result from ring stretching modes, that the band around $1300 \mathrm{~cm}^{-1}$ is dominated by $\mathrm{CC}$ stretching, and that numerous $\mathrm{CH}$ and $\mathrm{CCC}$ bending modes are present below $1200 \mathrm{~cm}^{-1}$. These assignments will be compared with the results of our calculations and discussed in more detail below.

The data in Fig. 1 clearly scales with the number of molecules available for scattering. As an example, the inset depicts the dependence of the Raman intensity of the $1603 \mathrm{~cm}^{-1}$ mode on the SP6 coverage. It shows that the scattering intensity scales linearly with the number of scatterers and therefore excludes non-linear effects. Remarkably, even the $1.0 \mathrm{~nm}$ film, which corresponds roughly to monolayer coverage, still exhibits an SP6 signal that is on the order of the $418 \mathrm{~cm}^{-1}$ mode of the sapphire (bulk) substrate, suggesting a high scattering efficiency of the SP6 molecules. Assuming a detection probability of $1 \%$ of scattered photons, we estimate a considerable (non-resonant) scattering cross section of $2.2 \cdot 10^{-25} \mathrm{~cm}^{2} / \mathrm{sc}$ for SP6.

The absence of a shift in the vibrational frequencies with coverage suggests relatively weak substrateadsorbate and inter-molecule interactions. ${ }^{18}$ Therefore, in order to identify the origin of the observed modes, we performed DFT calculations of the isolated molecule. The results are shown in Fig. 2 (bottom) and compared to the experimental Raman spectrum of an $8.0 \mathrm{~nm}$ film of SP6 on a sapphire substrate (top). As demonstrated in a previous publication ${ }^{2}$, the film corresponds to a few amorphous monolayers of SP6 molecules. Overall, experiment and calculation are seen to agree very well. However, despite the scaling of the calculated results (see above), the calculations still exhibit a blue shift of approximately 10 $\mathrm{cm}^{-1}$ with respect to experiment. We attribute this shift in part to missing effects of anharmonicity, the neglect of the quantum nature of the nuclei and the limited basis set. More important, though, is the possibility that in the thin film the molecules adopt a slightly different geometric structure than in the gas phase. $^{19}$ 
Table 1 lists all the significant calculated and experimentally observed modes of this work and compares them with selected literature values of related molecules. In agreement with the previous studies, the dominant peak above $1600 \mathrm{~cm}^{-1}$ is assigned to the symmetric stretching mode of the rings. The corresponding mode of the SP6 molecule is illustrated in the right inset of Fig. 2. The weak modes at low frequencies, below $1270 \mathrm{~cm}^{-1}$, arise primarily from in-plane $\mathrm{CH}$ and $\mathrm{CCC}$ bending modes and ring stretching modes in the different parts of the SP6 molecule. In particular, there is a perfect match of the breathing mode and $\mathrm{CH}$ bend of the terminal rings in SP6 biphenyl groups with the oligophenyls ${ }^{14}$ at $1004 \mathrm{~cm}^{-1}$ and $1042 \mathrm{~cm}^{-1}$, respectively. This suggests that these modes of the terminal rings are not significantly affected by exchanging the spirobifluorene between them with additional phenyl rings.

The $\mathrm{CH}$ and $\mathrm{CCC}$ bending modes of the fluorene groups of the SP6 at slightly higher frequency correspond nicely to the same modes of the isolated spirobifluorene molecule ${ }^{17}$. Also in agreement with the literature ${ }^{15-17}$, the band around $1300 \mathrm{~cm}^{-1}$ arises from various CC stretch vibrations within the SP6. The left inset of Fig. 2, for example, depicts the inter-ring stretching of the biphenyl groups, which was also identified in Ref. [15]. Naturally, we did not find a literature value for the wagging mode $\left(1370 \mathrm{~cm}^{-1}\right)$ of the bonds between the biphenyl and tert-butyl groups with the spirobifluorene.

We now turn to the analysis of the substrate influence on the Raman spectra of SP6. For this purpose, we first compare the data of the $6.4 \mathrm{~nm}$ SP6 film on sapphire to the Raman response of the bare substrate. (two bottom spectra of Fig. 3). In agreement with the literature, we do not find any significant Ramanactive optical phonon modes of the sapphire substrate above $800 \mathrm{~cm}^{-1}$. For reference, we show the highest substrate signal measured (below $500 \mathrm{~cm}^{-1}$, intensities unscaled). The SP6 molecules are obviously extremely good Raman scatterers. The molecules' vibrational response exceeds the bulk sapphire signal, even in this case with coverage of just few monolayers.

Fig. 3 also depicts the spectra of similar SP6 layers on the polar $\mathrm{ZnO}(000-1)$ and $\mathrm{ZnMgO}(000-1)$ surfaces to check for any substrate-induced changes of the SP6 response. We do not anticipate any additional response from the $\mathrm{ZnMgO}(000-1)$ sample in the range of interest $\left(>800 \mathrm{~cm}^{-1}\right)$. For the $\mathrm{ZnO}(000-1)$ 
substrate, we expect to observe a broad band between 1000 and $1200 \mathrm{~cm}^{-1} \cdot{ }^{20,21,22}$ On first inspection, all spectra in Fig. 3 are dominated by the high frequency ring stretch and the band of CC stretching modes around $1300 \mathrm{~cm}^{-1}$. None of the observed modes is frequency shifted with respect to the data of SP6 on the other substrates.

We test the absence of substrate influences on the Raman spectrum in Fig. 4: The topmost curve displays the Raman signal of a $1.0 \mathrm{~nm}$ SP6 layer on ZnO. If the Raman response of the SP6 is unchanged by adsorption on $\mathrm{ZnO}$, subtraction of the $1.0 \mathrm{~nm} \mathrm{SP} 6 / \mathrm{Al}_{2} \mathrm{O}_{3}(11-20)$ spectrum (middle) should yield the Raman spectrum of $\mathrm{ZnO}$. The resulting bottom trace indeed coincides with the Raman response of $\mathrm{ZnO}(000-1)$ in the literature. ${ }^{20-22}$ We subtract this substrate-induced background from the spectrum of 8.0 $\mathrm{nm} \mathrm{SP6/ZnO(000-1)} \mathrm{and} \mathrm{divide} \mathrm{it} \mathrm{by} 8$. The resulting trace (orange) coincides almost perfectly with the spectrum of $1.0 \mathrm{~nm} \mathrm{SP} 6 / \mathrm{Al}_{2} \mathrm{O}_{3}(11-20)$, ultimately showing that there is no influence of the substrate on the Raman response of SP6 and confirming that the electronic structure of SP6 is not perturbed by interaction with the substrate surface. Furthermore, the absence of spectral differences between monoand multilayer strongly suggests that SP6 does not form a first wetting layer that is different from the bulk neither in its geometric nor in its electronic properties.

\section{Summary and Conclusions}

We have performed a systematic study of various coverages of SP6, spanning the monolayer to multilayer regime, by non-resonant Raman spectroscopy on $\mathrm{ZnO}(000-1)$, $\mathrm{ZnMgO}(000-1)$, and $\mathrm{Al}_{2} \mathrm{O}_{3}(11-20)$ substrates and have also performed DFT calculations of the gas phase molecule. Due to the good match between experiment and calculation, we were able to identify the main modes in the SP6 Raman spectrum. The Raman response is dominated by the symmetric ring stretch and strongly resembles the Raman spectra of related molecules. In particular, the spectrum exhibits the characteristic $\mathrm{CH}-$ and $\mathrm{CCC}$ bending modes as well as the CC-stretch vibration in the different parts of the molecule.

The high quality of the samples is indicated by the similarity of all Raman spectra, the linear dependence of the peak intensities on the number of molecules, and simple background subtraction. The large 
scattering cross section of the SP6 molecule, which offers Raman intensities on the order of the substrate signal, enabled the comparison of Raman spectra of SP6 on different substrates even for monolayer coverages. This comparison unambiguously showed that adsorption of SP6 has no meaningful impact on the vibrational response of the molecule. We therefore conclude that SP6 adsorbs without any significant electronical perturbation on all of the investigated surfaces.

\section{Acknowledgements}

We are grateful to Katrin F. Domke and Martin Wolf for helpful comments and discussions. This work was partially funded by the German Science Foundation (DFG) through Sfb 951. J.S. acknowledges support from the Alexander von Humboldt Foundation. Raman spectroscopy measurements at Columbia University were supported by the Center for Re-Defining Photovoltaic Efficiency through MolecularScale Control, an Energy Frontier Research Center funded by the U.S. Department of Energy (DOE), Office of Science, Office of Basic Energy Sciences under Award DE-SC0001085. 


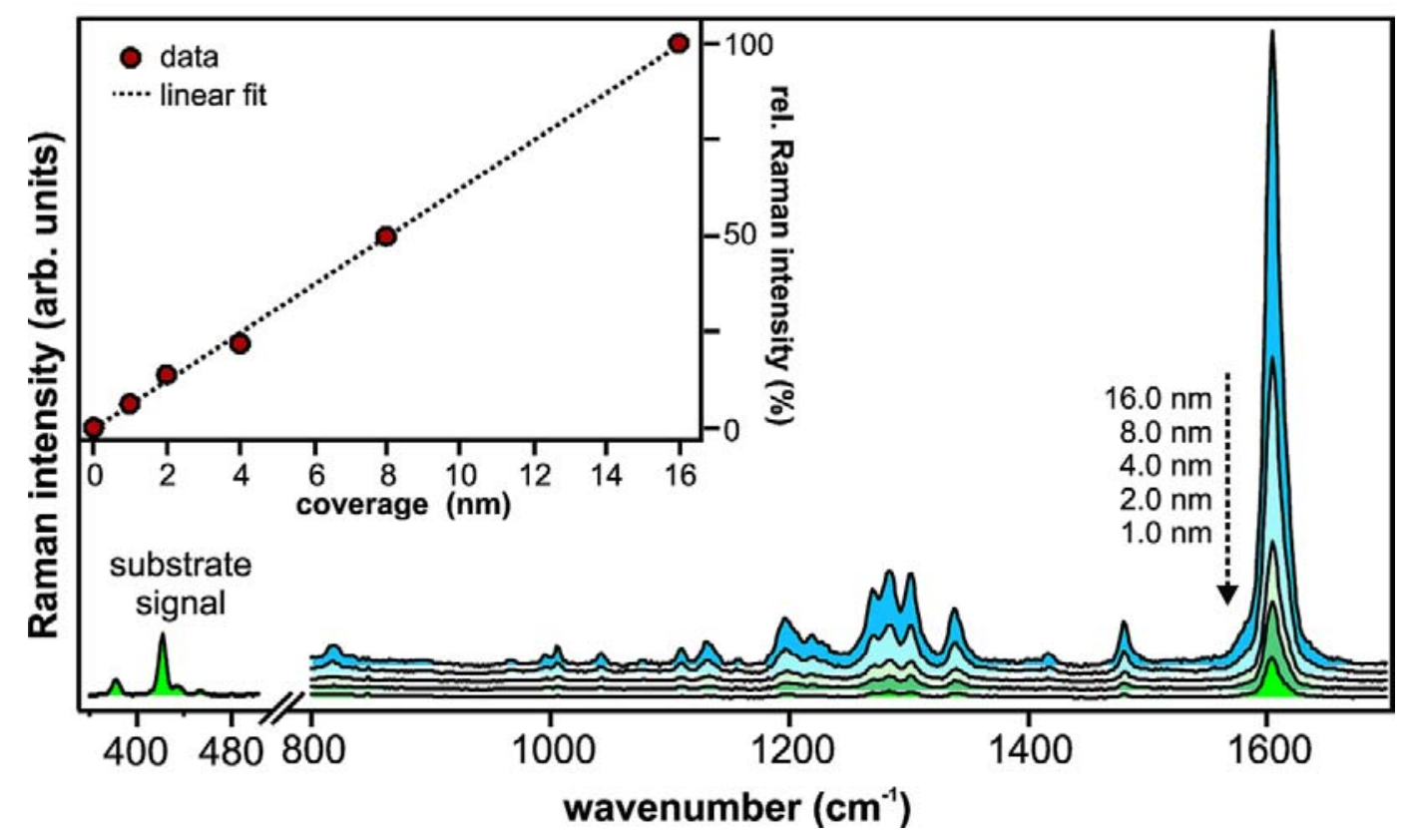

Fig. 1: Coverage dependence of the SP6 Raman response. Films were deposited on a sapphire substrate and span the monolayer to multilayer range. Inset: The Raman intensity scales with the number of molecules in the organic film. Error bars lie within the markers. 


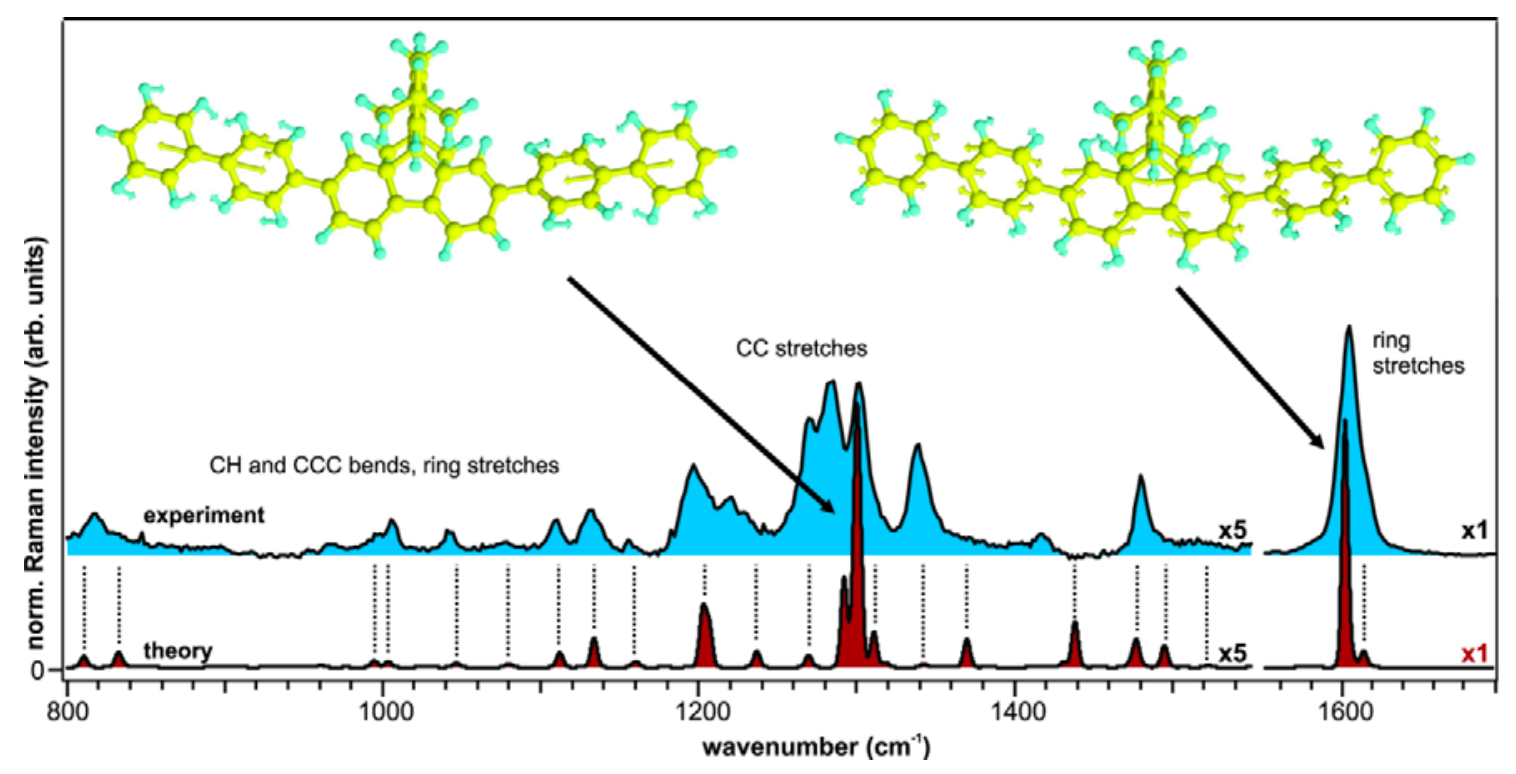

Fig. 2: Calculated Raman spectrum (bottom, artificial broadening of $2 \mathrm{~cm}^{-1}$ ) in comparison to the experimental Raman scattering intensities of an $8.0 \mathrm{~nm}$ film of SP6 on sapphire (top). The calculated spectrum has been shifted to lower frequencies by $10 \mathrm{~cm}^{-1}$ in order to match the experimental modes (see text for details). Insets: Inter-ring CC stretch of the biphenyl groups at $1300 \mathrm{~cm}^{-1}$ (left) and the symmetric ring stretch at 1602 $\mathrm{cm}^{-1}$ (right). 


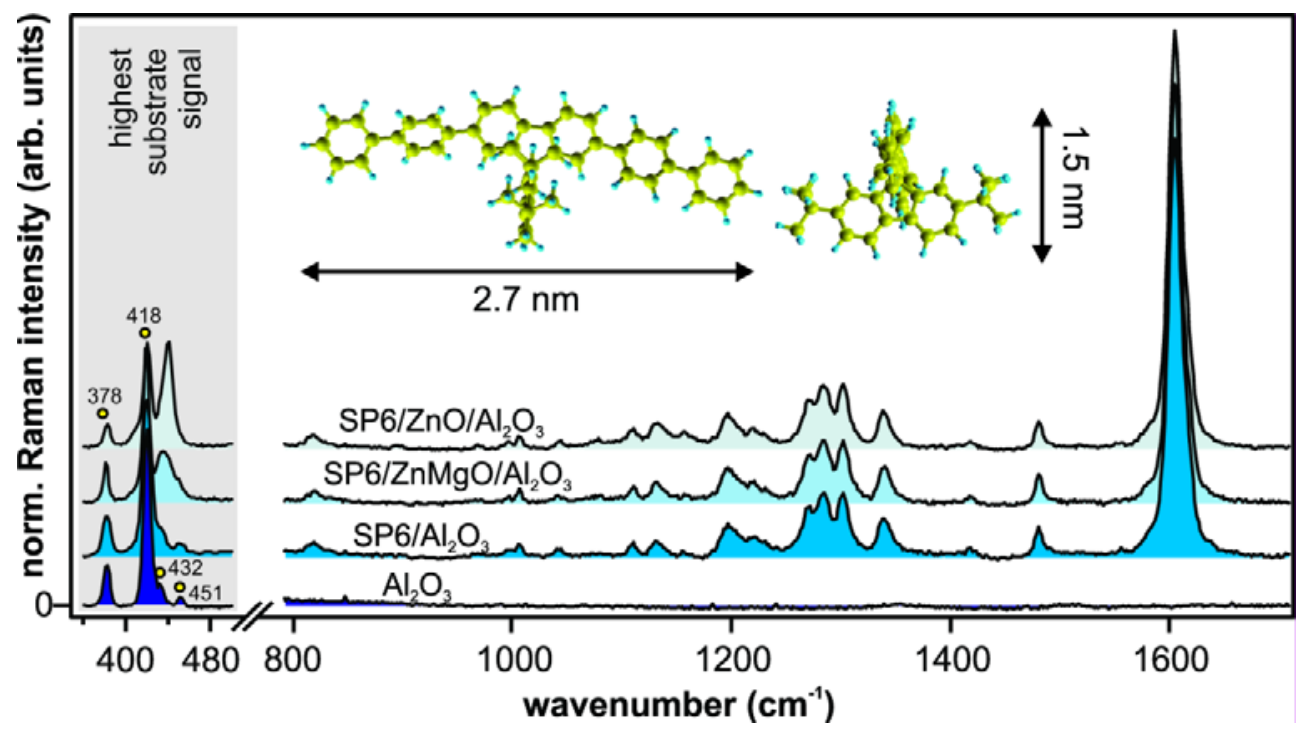

Fig. 3: Raman spectra of $8.0 \mathrm{~nm}$ SP6 on sapphire, $\mathrm{ZnMgO}(000-1)$, and $\mathrm{ZnO}(000-1)$. The bottom spectrum depicts the sapphire response for comparison, referenced to literature values taken from Ref. [23] (yellow markers). The signal at low frequencies results from the respective substrates only. Inset: SP6 molecule as calculated by DFT. 


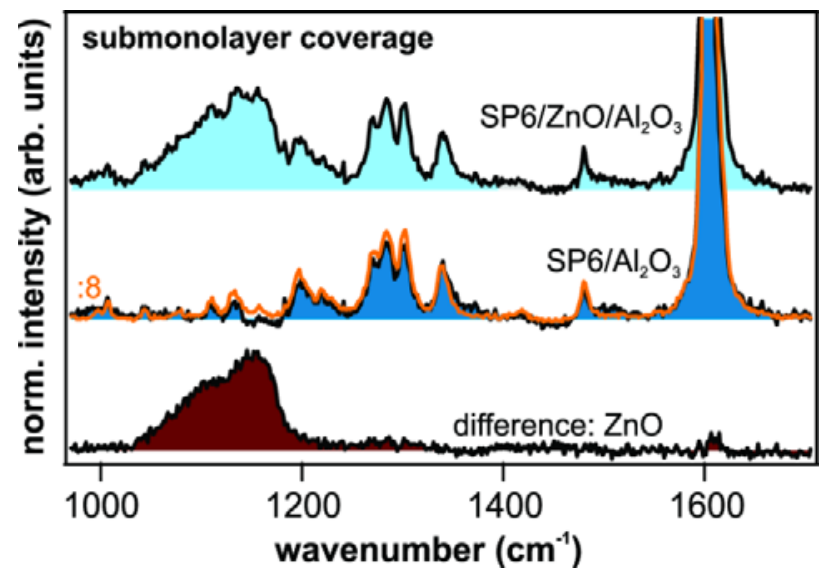

Fig. 4: Comparison of $1.0 \mathrm{~nm} \mathrm{SP} 6$ (mass equivalent) on $\mathrm{ZnO}(000-1)$ and sapphire showing that there is no substrate-induced difference in the response of the molecules. The band between 1050 and $1200 \mathrm{~cm}^{-1}$ results from phonons in the $\mathrm{ZnO}(000-1)$ film. The signal of $8.0 \mathrm{~nm}$ SP6 on $\mathrm{ZnO}(000-1)$ matches the $1.0 \mathrm{~nm} \mathrm{SP} 6 / \mathrm{Al}_{2} \mathrm{O}_{3}(11-20)$ trace nicely after subtraction of the $\mathrm{ZnO}(000-1)$ signal and scaling by a factor of 8 (orange curve). 


\begin{tabular}{|c|c|}
\hline$v\left(\mathrm{~cm}^{-1}\right)$ & $\begin{array}{l}\text { literature } \\
\text { approx. type }^{\mathrm{a}}\end{array}$ \\
\hline $\begin{array}{c}992-6^{[\mathrm{e}]} \\
1004-5^{[\mathrm{e}]} \\
1020^{[\mathrm{b}]} \\
1039-42^{[\mathrm{e}]}\end{array}$ & $\begin{array}{l}\text { ip def term rings }(p-\mathrm{P}) \\
\text { ip def term rings }(p-\mathrm{P}) \\
\text { ip CH \& CCC bend (SBF) } \\
\text { ip CH bend terminal rings }(p-\mathrm{P})\end{array}$ \\
\hline $1105^{[\mathrm{b}]}$ & ip $\mathrm{CH} \& \mathrm{CCC}$ bend (SBF) \\
\hline $1148^{[\mathrm{b}]}$ & ip $\mathrm{CH} \& \mathrm{CCC}$ bend (SBF) \\
\hline $\begin{array}{l}1221^{[\mathrm{b}]} \\
1255^{[\mathrm{d}]} \\
1275-9 \\
1280^{[\mathrm{d}]}\end{array}$ & $\begin{array}{l}\text { ring str, ip CH bend (SBF) } \\
\text { ir CC str (F8-oligomers) } \\
\text { ir CC str ( } p \text {-P) } \\
\text { ir CC str (F8-oligomers) }\end{array}$ \\
\hline $1288^{[\mathrm{b}]}$ & ip $\mathrm{CH}$ and $\mathrm{CCC}$ bend (SBF) \\
\hline $\begin{array}{l}1303^{[\mathrm{c}]} \\
1305^{[\mathrm{d}]}\end{array}$ & $\begin{array}{l}\text { ir CC str (BP) } \\
\text { ir CC str (F8-oligomers) }\end{array}$ \\
\hline $\begin{array}{l}1345^{[\mathrm{d}]} \\
1351^{[\mathrm{c}]} \\
1354^{[\mathrm{b}]}\end{array}$ & $\begin{array}{l}\text { ir CC str (F8-oligomers) } \\
\text { ir CC str (F) } \\
\text { ip CH \& CCC bend (SBF) }\end{array}$ \\
\hline $\begin{array}{l}1444^{[\mathrm{b}]} \\
1471^{[\mathrm{b}]}\end{array}$ & $\begin{array}{l}\text { ip } \mathrm{CH} \& \mathrm{CCC} \text { bend }(\mathrm{SBF}) \\
\text { ip } \mathrm{CH} \& \mathrm{CCC} \text { bend (SBF) }\end{array}$ \\
\hline $\begin{array}{c}1573^{[\mathrm{b}]} \\
1593-5^{[\mathrm{e}]} \\
1596^{[\mathrm{b}]} \\
1604-5^{[\mathrm{e}]} \\
1614^{[\mathrm{c}]}\end{array}$ & $\begin{array}{l}\text { ip } \mathrm{CH} \& \mathrm{CCC} \text { bend (SBF) } \\
\text { inner ring str }(p-\mathrm{P}) \\
\text { ip } \mathrm{CH} \& \mathrm{CCC} \text { bend (SBF) } \\
\text { term ring str }(p-\mathrm{P}) \\
\text { sym ring str }(\mathrm{BP})\end{array}$ \\
\hline
\end{tabular}

\begin{tabular}{|c|c|c|}
\hline $\begin{array}{l}\text { exp. } \\
v\left(\mathrm{~cm}^{-1}\right)\end{array}$ & $\begin{array}{l}\text { theo. } \\
v\left(\mathrm{~cm}^{-1}\right)\end{array}$ & $\begin{array}{l}\text { our work } \\
\text { approx. type }^{\text {a }}\end{array}$ \\
\hline 819 & 810 & ip ring str $(\mathrm{F} 2)$ \\
\hline 833 & 833 & ip ring str (F2) \\
\hline 996 & 995 & ip CCC bend (spiro) \\
\hline 1006 & 1004 & ip breathing term rings $(\mathrm{BP})$ \\
\hline-- & -- & \\
\hline 1042 & 1046 & ip $\mathrm{CH} \& \mathrm{CCC}$ bend (BP) \\
\hline 1077 & 1080 & ip $\mathrm{CH}$ bend (di-tert-butylfluorene) \\
\hline 1110 & 1112 & ip $\mathrm{CH}$ bend (F2) \\
\hline 1131 & 1133 & ip in phase $\mathrm{CH} \& \mathrm{CCC}$ bend (SBF) \\
\hline 1155 & 1160 & ip out of phase $\mathrm{CH} \& \mathrm{CCC}$ bend (SBF) \\
\hline 1195 & 1203 & ip in phase sym $\mathrm{CH}$ bend (BP) \\
\hline 1220 & -- & \\
\hline-- & 1237 & ip $\mathrm{CH}$ bending modes \\
\hline 1270 & 1270 & ip sym $\mathrm{CH}$ bend (F1) \\
\hline-- & -- & \\
\hline 1285 & 1292 & $\begin{array}{l}\text { ip in phase sym CC str (TB) } \\
\text { ip } \mathrm{CH} \& \mathrm{CCC} \text { bend (F2) }\end{array}$ \\
\hline 1302 & 1300 & ip ir in phase CC str (BP) \\
\hline 1314 & 1311 & ip CC str \& $\mathrm{CH}$ bend (F1) \\
\hline 1339 & 1343 & ip CC str \& $\mathrm{CH}$ bend (F2) \\
\hline-- & -- & \\
\hline-- & 1370 & ip CC wagging (BP\&F1, TB\&F2 bond) \\
\hline 1417 & $\begin{array}{c}-- \\
1448\end{array}$ & ip asym CC str, $\mathrm{CH}$ bend (F2) \\
\hline 1480 & 1479 & ip asym $\mathrm{CC}$ str, $\mathrm{CH}$ bend (F1) \\
\hline-- & 1495 & ip $\mathrm{CH}$ bend (fluorene-bis-biphenyl) \\
\hline 1582 & 1578 & ip str \& $\mathrm{CH}$ bend (F1) \\
\hline 1605 & $\begin{array}{l}1602 \\
1614\end{array}$ & $\begin{array}{l}\text { ip sym ring str (all rings) } \\
\text { ip asym ring str (all rings) }\end{array}$ \\
\hline
\end{tabular}

Table 1: Comparison of the calculated and measured Raman responses of the SP6 molecule from this work (right) with Raman modes of related molecules from the literature. ${ }^{a}$ ip $=$ in plane, sym $=$ symmetric, asym $=$ antisymmetric, str $=$ stretch, ir $=$ inter ring, def $=$ deformation, term $=$ terminal, $\mathrm{TB}=$ tert-butyl, F1 = SP6 fluorene between the biphenyls, F2 = SP6 fluorene with tert-butyl groups ${ }^{\mathrm{b}}$ spirobifluorene (SBF) modes from Ref. [17], ${ }^{\mathrm{c}}$ biphenyl (BP) and fluorene (F) modes from Ref.[15], ${ }^{\mathrm{d}}$ octylfluorene (F8) oligomer modes from Ref. [16], ${ }^{\mathrm{e}} p$-oligophenyl (p-P) modes from Ref. [14] 
${ }^{1}$ V.M. Agranovich, Y.N. Gartstein, and M. Litinskaya, Chem. Rev. 111, 5179 (2011)

${ }^{2}$ S. Blumstengel, S. Sadofev, C. Xu, J. Puls, R. L. Johnson, H. Glowatzki, N. Koch, and F. Henneberger, Phys. Rev. B 77, 085323 (2008)

${ }^{3}$ C. C. Neacsu, J. Dreyer, N. Behr, and M. B. Raschke Phys. Rev. B 73, 193406 (2006)

${ }^{4}$ K. F. Domke and B. Pettinger Phys. Rev. B 75, 236401 (2007)

${ }^{5}$ C. C. Neacsu, J. Dreyer, N. Behr, and M. B. Raschke, Phys. Rev. B 75, 236402 (2007)

${ }^{6}$ K. F. Domke, D. Zhang, and B. Pettinger J. Phys. Chem. C 111, 8611 (2007)

${ }^{7}$ H. Liang, H. Tian, and R. L. McCreery Appl. Spec. 61, 6, 613 (2007)

${ }^{8}$ B. Giese and D. McNaughton J. Phys. Chem. B 106, 1461 (2002)

${ }^{9}$ S. Sadofev, S. Blumstengel, J. Cui, J. Puls, S. Rogaschewski, P. Schafer, Y.G. Sadofyev, and F. Henneberger, Appl. Phys. Lett. 87, 091903 (2005).

${ }^{10}$ Gaussian 09, Revision A.02, M. J. Frisch, G. W. Trucks, H. B. Schlegel, G. E. Scuseria, M. A. Robb, J. R. Cheeseman, G. Scalmani, V. Barone, B. Mennucci, G. A. Petersson, H. Nakatsuji, M. Caricato, X. Li, H. P. Hratchian, A. F. Izmaylov, J. Bloino, G. Zheng, J. L. Sonnenberg, M. Hada, M. Ehara, K. Toyota, R. Fukuda, J. Hasegawa, M. Ishida, T. Nakajima, Y. Honda, O. Kitao, H. Nakai, T. Vreven, J. A. Montgomery, Jr., J. E. Peralta, F. Ogliaro, M. Bearpark, J. J. Heyd, E. Brothers, K. N. Kudin, V. N. Staroverov, R. Kobayashi, J. Normand, K. Raghavachari, A. Rendell, J. C. Burant, S. S. Iyengar, J. Tomasi, M. Cossi, N. Rega, J. M. Millam, M. Klene, J. E. Knox, J. B. Cross, V. Bakken, C. Adamo, J. Jaramillo, R. Gomperts, R. E. Stratmann, O. Yazyev, A. J. Austin, R. Cammi, C. Pomelli, J. W. Ochterski, R. L. Martin, K. Morokuma, V. G. Zakrzewski, G. A. Voth, P. Salvador, J. J. Dannenberg, S. Dapprich, A. D. Daniels, O. Farkas, J. B. Foresman, J. V. Ortiz, J. Cioslowski, and D. J. Fox, Gaussian, Inc., Wallingford CT (2009)

${ }^{11}$ A. P. Scott and L. Radom, J. Phys. Chem. 100, 41, 16502 (1996)

${ }^{12}$ Optical absorption of SP6 sets in above $3.2 \mathrm{eV}$ (387 nm, see Ref.[2]).

${ }^{13}$ This is the relevant spectral window. At lower frequencies $\left(200-800 \mathrm{~cm}^{-1}\right)$, we find a few substrate-induced modes, the highest is shown in Fig. 1; no signal is observed at higher frequencies $\left(1700-2000 \mathrm{~cm}^{-1}\right)$ (not shown).

${ }^{14}$ H. Ohtsuka, Y. Furukawa, M. Tasumi. Spectrochim. Acta 49A, 5/6, 731 (1993)

${ }^{15}$ F. Negri and M. Z. Zgierski. J. Chem. Phys. 97, 7124 (1992)

${ }^{16}$ W. C. Tsoi and D. G. Lidzey. Phys. Condens. Matter 20, 125213 (2008)

${ }^{17}$ B. H. Boo, J. Park, H. G. Yeo, S. Y. Lee, C. J. Park, and J. H. Kim. J. Phys. Chem. A 102, 1139 (1998)

${ }^{18}$ Small line shifts on the order of the line width might be hidden due to line-broadening effects.

${ }^{19}$ Biphenyl, for instance, exhbits a dihedral angle of ca. $44^{\circ}$ in the gas phase but close to $0^{\circ}$ in the crystal. Pressure dependent studies on biphenyl showed that the planarization can induce a significant shift in the biphenyl-stretch modes. At the same time, the planarization also led to a significant increase of the Raman intensity of the molecule. See M. Zhou, K. Wanga, Z. Mena, S. Gaoa, Z. Liaand C. Sun. Spectrochim. Acta A 97, 526 (2012).

${ }^{20}$ B. N. Mavrin, L. N. Demyanets, R. M. Zakalukin. Phys. Lett. A 374, 4054 (2010)

${ }^{21}$ L. J. Wang, G. J. Exarhos. Thin Solid Films 519, 1495 (2010) 
${ }^{22}$ M. Schumm. ZnO-based semiconductors studied by Raman spectroscopy: semimagnetic alloying, doping, and nanostructures, dissertation (2009), http://opus.bibliothek.uni-wuerzburg.de/volltexte/2009/3704/

${ }^{23}$ M. C. Munisso, W. Zhu, and G. Pezzotti. Phys. Status Solidi B 246, 1893 (2009) 